\title{
THE TUMORIGENIC ACTION OF BETA, PROTON, ALPHA AND ELECTRON RADIATION ON THE RAT SKIN
}

Progress Report

Roy E. Albert and Fredric J. Burns

Institute of Environmental Medicine New York University Medical Center New York, N.Y. 10016

August 1, 1973 - July 31, 1974

PREPARED FOR THE U. S. ATOMIC ENERGY COMMISSION UNDER CONTRACT NO. AT(1I-1) 3380

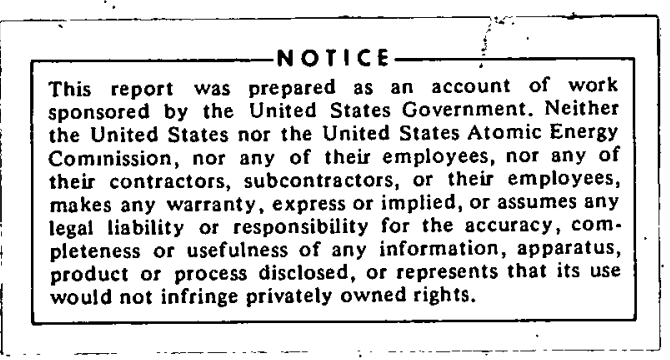




\section{DISCLAIMER}

This report was prepared as an account of work sponsored by an agency of the United States Government. Neither the United States Government nor any agency Thereof, nor any of their employees, makes any warranty, express or implied, or assumes any legal liability or responsibility for the accuracy, completeness, or usefulness of any information, apparatus, product, or process disclosed, or represents that its use would not infringe privately owned rights. Reference herein to any specific commercial product, process, or service by trade name, trademark, manufacturer, or otherwise does not necessarily constitute or imply its endorsement, recommendation, or favoring by the United States Government or any agency thereof. The views and opinions of authors expressed herein do not necessarily state or reflect those of the United States Government or any agency thereof. 


\section{DISCLAIMER}

Portions of this document may be illegible in electronic image products. Images are produced from the best available original document. 


\section{Progress Report}

\subsection{General Summary of the Project}

As a means of gaining a better understanding of the nature of the carcinogenic hazard of radiation, the mechanism of cancer induction has been studied by irradiating rat skin in various geometrical and temporal patterns with various types of radiation. One of the initial questions was whether the incidence of tumors was proportional to the volume of irradiated tissue. The results of various sieve and grid irradiation patterns has indicated that focal irradiation is less efficient in producing tumors than uniform irradiation for low LET radiation, such as electrons or $\mathrm{x}$-rays, while no difference in efficiency was observed for protons. By varying the energy of an electron beam, and hence its penetration, it was found that tumors were produced only if the radiation penetrated sufficiently to reach the level of about $0.3 \mathrm{~mm}$ which roughly corresponded to the depth of the hair follicles in the resting (telogen) phase. Several experiments have been conducted and are continuing which are directed at elucidating the biological basis for the penetration effect. In. relation to the cellular mechanisms involved in radiation carcinogenesis, comparisons have been made of the carcinogenic impact of proliferation at the time of and subsequent to irradiation and the relationship of tumor growth rate to appearance 
time has been studied. Another broad segment of the program has focused on the recovery processes for tumor induction inherent in the results of fractionation experiemtns where recovery as a function of time between fractions can be used to evaluate the dose rate effect in radiation carcinogenesis.

\subsection{Progress Achieved in the Preceding Year}

The major goals for the year have been achieved although there have been some priority adjustments in the sense that certain areas have been developed more slowly while others have been developed more quickly than originally anticipated. The following is a point by point status report for each section in last year's proposal.

2.1 Postirradiation Proliferation and Tumor Development This was a comprehensive experiment which sought to establish quantitatively. how proliferation rate in the hair follicle and epidermal cell populations at the time of and subsequent to irradiation affected the number and appearance time of the skin tumors. The results indicate that sustained follicular proliferation induced by repeatedly plucking the hair has little or no effect on the tumor response pattern. Repeated stimulation of the epidermis by surface abrasion had either no' effect (at low doses) or reduced the rate of tumor appearance (at high doses). 
2.2 Early Mörphological Response of Growing Hair Follicles to Various Penetrations

The penetration effect on the morphology of the growing hair follicle has been prepared in the form of a preprint that is submitted here as AEC 3380-12. Total cellularity, total DNA and the specific activity of the DNA were measured at various times after irradiation for several doses and penetrations. The basic question was whether the entire growing hair follicle, which is. a large, mitotically active structure, needs to be irradiated in order to produce permanent morphological injury. A second question was to what extent the rapid proliferation at the time of irradiation might accelerate repopulation and repair in the early stages of the injury response. The results were to be compared to the tumor response pattern for similar doses and penetrations.

Permanent morphological injury to the hair follicles was produced most efficiently when substantial doses were delivered at least to the level of the follicle matrix at about 0.8 to $1.0 \mathrm{~mm}$. There was evidence that the morphological injury developed slowly possibly requiring at least several weeks. No evidence for repopulation or regeneration was found in the initial 4 days. postirradiation in spite of considerable loss of cells in the 
various epithelial compartments of the skin. Repopulation did occur in epidermis and sebaceous glands often so vigorously that hyperplasia developed and sometimes persisted for many months. A type of hyperplasia was noted in the hair follicles where the growing phase was prolonged in some follicles well beyond the time noted in control rats.

\subsubsection{The Penetration Effect for Tumor Induction in Growing Phase Skin}

- As already noted in section 2.2, the hair. follicle in the growing stage is much deeper in the skin and more mitotically active than the resting follicle. It was of interest to determine whether there was a minimum penetration requirement for skin tumor induction in growing phase and, if so, whether its value corresponded to the depth found for resting phase follicles, i.e., at the level of the germ cells, or was deeper corresponding with the actual depth of the follicle. A separate question was whether for a given dose distribution the mitotically active structure would be inherently more sensitive to the carcinogenic action of radiation or not. Several doses were given at penetrations of $2.0 \mathrm{~mm}, 1.5 \mathrm{~mm}, 1.0 \mathrm{~mm}$ and $0.5 \mathrm{~mm}$.

The tumor response was consistent with the assumption that a minimum penetration approximately the same as in resting phase was necessary in order to produce tumors. In 
other words, the sensitive site for tumor induction remained in a superficial location in spite of the increased size and depth of the growing follicle.

For shallow penetrations, such as $1.0 \mathrm{~mm}$, a peak tumor yield occurred well below the dose for onset of ulceration which suggests that tumor sensitive cells may be killed even'at doses that are not sufficiently penetrating to cause gross destruction of the skin and hair follicles. These results raise the interesting question of what the target cell population might be and under what conditions radiation overdose might lead to their destruction.

\subsection{Tumor Growth Rate and Appearance Time (AEC-3380-13)}

A single dose of radiation in rat skin produces tumors that appear at a more or less constant rate (after an initial tumor free interval) throughout the animal's life. A question arises whether all the tumors actually started to grow as cell masses at the time of irradiation and appearance time is determined by growth rate or new tumors are constantly appearing and growth is independent of time. An initial study indicated that growth rate distributions were independent of the time of initial appearance, but the growth rates were so variable from tumor to tumor that the conclusions could have been compromised. Consequently, a study was launched to obtain detailed 
information regarding the growth rate characteristics of tumors in the size range above $1.0 \mathrm{~mm}$ in diameter and a special technique was developed to detect tumors as small as $0.1 \mathrm{~mm}$ in diameter. The growth rate analysis indicated that most of the tumors underwent retarded growth, i.e., growth rate declined with increasing size, and some reached asymptotic sizes. The search for microtumors indicated far fewer than expected on the basis of the assumption that all tumors were present from the time of irradiation. Once the tumors were large enough to detect, the incidence curves were displaced in a parallel manner for various size endpoints indicating that the major events affecting appearance time occurred at a stage of tumor development prior to detection, even as a microtumor. The results were consistent with the assumption that new tumors were forming constantly throughout the animal's 1 ife.

In spite of the necessity to irradiate to at least $0.3 \mathrm{~mm}$ to produce tumors, about $75 \%$ of the microtumors small enough to be assigned a definite location were in the outermost. $0.15 \mathrm{~mm}$, i.e., essentially in or close to the epidermis. The implications of the above finding are to be explored more fully in future work.

2.4 Fractionation of Cyclotron Accelerated Protons and Tumor Induction

In cooperation with Drs. Kuo and Laughlin 
at the sloan Kettering Institute in New York, rats were irradiated with $15 \mathrm{MeV}$ protons in order to determine the degree of recovery for tumor induction. Various doses (from 150 rads up to 1500 rads) were given either as single doses or 24 hrs after a uniform dose of 300 rads. The experiment, consisting of 14 dose groups of 20 rats per group, is currently in progress and only the very earliest indication is that recovery did occur. Protons have an intermediate value of linear energy transfer (LET) and it would be important to determine possible recovery and dose rate effects because protons have a relatively high relative biological effectiveness (RBE).

\subsubsection{The Effect of Proton Fractionation on Hair Follice Survival (AEC 3380-14)}

One of the graduate students on the project compared single and fractionated proton doses with respect to the ability of the follicle to respond to growth stimulation and to produce hair. His technique consisted of a histological determination of the ability of the hair follicle cells to proliferate and produce a mature growing follicle at various times after irradiation. The proton results were then compared to comparable results for electrons. The results indicated that while protons were about 2 times more efficient than electrons in producing hair follicle effects, nevertheless, measurable recovery occurred comparable to about $60 \%$ of the initial dose. Eventually 
a comparison between the early hair follicle effects and the tumor incidence will be made.

\subsection{Model of the Dose Rate Effect in Radiation Carcinogenesis}

Some theoretical, work has been completed on a dose rate model based on the assumption that tumor induction requires two. sequential stages, the first of which is recoverable. In the model the dose rate effect is completely predictable if the effects of multiple single doses are additive and if the recovery rate is known. The recovery rate is measurable in fractionation experiments which are described separately below. The mathematical formalism leads to expressions for tumor appearance rate as a function of the total dose for any dose rate. For exposure times that extend for a considerabel proportion of an animal's life, it must be assumed that there is little, if any, dependence of susceptibility on age. The model is being extended to include estimates of final tumor incidence from the experimental functions relating incidence and time for single exposures.

\subsection{Carcinogenic Response as a Function of Age}

An experiment to evaluate the carcinogenic sensitivity of rat skin as a function of age at the time of irradiation was begun: Newborn rats were irradiated with Grenz rays (low voltage $\mathrm{x}$-rays) at various doses from $500 \mathrm{R}$ to $3000 \mathrm{R}$. In the newborns the hair follicles were in early anagen, although hair had not yet 
erupted above the surface. The newborns will be observed for tumors and eventually a comparison of tumor response in older. rats in the same stage of the hair cycle will be made. An immediate comparison will be possible with 28 day old rats and 58 day old rats from previous experiments by making adjustments in the data to compensate for the hair cycle effect.

2.7 Kinetics of the Fractionation Effect for Tumor Induction The dose rate effect in radiation carcinogenesis is related to recovery processes that, in principle, are measurable in fractionation experiments. By varying the time interval between fractions, the recovery rate constant can be measured. The present experimen't is an attempt to measure the recovery rate constant for tumor induction in rat skin and to determine whether the constant is dose dependent.

The single dose response curve for tumor induction will be determined at the following doses: $500,1000,750,1250,1500$ and 2500 rads. At 500,750 and 1250 rads, two fractions were given separated by $0.25,1,3$ and 6 hours. There were approximately 20 rats in each experimental group. Some sections of the experiment were begun about 4 months ago while other sections were begun as recently as 1 month ago. So far no definitive tumor data is available. Preliminary indications based on the acute skin reaction suggest a recovery halftime between 2 and 3 hours. 
2.8 Carcinogenic Interaction of Ionizing Radiation and Ultraviolet Light

The u?traviolet light experiments have not progressed as rapidly as originally anticipated. However, the ultraviolet irradiation and detection equipment will soon be operational and some experiments will be under way, by the end of the contract year. Part of the justification for investigating the carcinogenic interaction of ultraviolet light and ionizing radiation is that human skin is known to be sensitive to ultraviolet carcinogenesis, and it would be important to know about possible interactions with ionizing radiation. Another part of the justification for investigating the interaction is based on the interesting physical and biological properties of ultraviolet radiation. In mouse skin broad spectrum ultraviolet from a sun lamp accelerates the growth of tumors and it would be important. to determine if the accelerating activity extends to tumors induced by ionizing radiation. Short wavelength ul-raviolet is absorbed so strongly in the epidermis that virtually none penetrates as far as the dermal tissue thus by selectively irradiating epidermis it will be possible to investigate lethality and regeneration in relation to the site of origin of tumors induced by ionizing radiation. Initial experiments will focus on mouse skin with extension to rat skin in order to answer specific questions in that system. 
2.9 Growth Fraction and the Go-Phase in the Cell Cycle

A paper was presented at the 13th Annual Hanford Biology Symposium which described certain theoretical aspects of the growth fraction concept and how it relates to the $G_{O}$-phase. Labeled mitosis curves for a variety of tumors and normal tissues were fitted to the cruves predicted by the model with good agreement. The $G_{0}$-phase was most pronounced in slowly growing autochthonous tumors and was essentially absent in rapidly growing transplantable tumors. The growth fraction varied over wide limits essentially independent of the growth rate and type of tumor.

2.10 The Sieve Effect for Cell Survival in Plateau Phase Cells in Tissue culture

As discussed above, a reduction in tumor yield per unit area in rat skin was noted for low LET (linear energy transfer) radiation when applied in a sieve pattern in comparison to a uniform dose distribution. A pilot study has been undertaken by one of the graduate students working on the project to determine whether sieve protection occurs for cell survival in a tissue culture system. Currently, baseline dose-response curves are being established for a line of mouse fibroblast cells (Balb/C 3T3) and techniques are being developed for assaying cell survival as a function of distance from a line demarcating the interface 


\subsection{Cell Kinetics and Tumor Induction in Rat Liver for Various Dietary. Carcinogens}

As a neans of contrasting the tumor response in a diverse organ, such as liver, with skin, investigations have been carried out to determine the relationship of hepatic cell loss and proliferation and tumor incidence to time during and after continuous dietary levels of various carcinogens, including 2fluorenylacetamide (2-FAA), diethylnitrosamine (DEN) and aflatoxin. For 2-FAA early cell loss was followed by apparent regeneration while at the same carcinogenic dose DEN produced hyperplasia without signitifcant cell loss. The enhanced proliferation and hyperplasia of the DEN response was reversible and the tissue returned to apparently normal levels when the DEN was stopped. Hepatomas appeared even when the DEN was stopped early although they were delayed in comparison to continuous DEN. These studies are being extended to include higher doses of aflatoxin.

\subsection{Bibliography}

3380-1l Burns, F. J., R. E. Albert and I. P. Sinclair. The effect of a 24 hour fractionation interval on the induction of rat skin tumors by electron radiation. Radiat. Res. (submitted)

3380-12 Sinclair, I. P., F. J. Burns and R. E. Albert. The response of growing hair follicles to various penetrations of electron radiation in rat skin. Radiat. Res. (manuscript) 
3380-13 Burns, F. J., M. Vanderlaan and R. E. Albert. Growth rate and appearance time of radiation-induced rat skin tumors. Fifth ICRR (Conference Report), 1974.

3380-14 Strickland, P. The effect of fractionated doses of protons and electrons on hair follicles in the albino rat. (Masters thesis)

3380-15 Burns, F. J. Theoretical Aspects of growth fraction in a. Go model. Hanford Biology Symposium (Conference Report), 1973 .

3380-16 Progress Report for year ending July 31, 1974 . 